\title{
Highlights from this issue
}

Robert Scott-Jupp, Acting Editor-in-Chief

\section{An unusual randomised} controlled trial on a hot topic

It is rare in clinical journals to see randomised controlled trials (RCTs) where the intervention is applied to whole communities rather than individual patients. Edwards et al randomised clusters of homes in London social housing estates which shared central hot water supplies. They aimed to address the issue of whether it is possible to reduce the temperature of hot tap water to $<60^{\circ} \mathrm{C}$ and thereby reduce risk of scalds, while centrally heating the water overnight sufficiently to prevent Legionella colonisation. They found that it was, at least in terms of their outcomes of measured water temperatures. This study was clearly not designed to detect differences in either scald rates or Legionella infection. This paper neatly complements one we carried in March which demonstrated the effectiveness of thermostatic mixer valves in reducing water temperatures to a safe level. ${ }^{1}$ One or other of these measures could be applied to every household.

Scalds remain the commonest cause for admission to children's burns units in the UK, and are more common in socially deprived families. ${ }^{2}$ Simple interventions such as this are likely to be more effective than education campaigns. See page 1097.

\section{Imaging in suspected abusive head trauma}

Diagnosing non-accidental injury (NAI) is always difficult, but perhaps never more so than when confronted, unexpectedly, with an infant with an abnormal brain scan and no convincing history to explain it. Kemp et al from the Welsh Child Protection Systematic Review Group have produced a characteristically thorough review to help us. Many of these cases have no satisfactory outcome in that doubt remains as to whether abuse occurred. They restricted their data collection to those cases where the diagnosis of NAI was as certain as it could be. This allowed them to identify specific neuroradiological features - hypoxicischaemic injury, oedema and subdural haemorrhages, particularly if multiple and in certain locations - that are associated with abusive trauma. Most of the children in the 21 studies they analysed had only CT rather than MRI scans. As MRI is being increasingly used in these cases, in the future its superior imaging detail should make it easier to discriminate. See page 1103.

\section{Drug therapy: something for everyone}

This month's Drug Therapy section has articles of interest to all paediatricians and parents. Everybody knows that fever is bad, that paracetamol and ibuprofen make it better, and that the two together make it better still: but is it, and do they? Purssell's systematic review finds very little evidence that giving combined antipyretic treatment has any advantage over giving a single drug, at least in terms of short-term, clinically significant outcomes. He thus elegantly contradicts what is everyday practice in most hospitals, practices and homes in the UK, and, I suspect, elsewhere. He also questions the need to treat fever at all when it is causing no discomfort. Unusually for a systematic review, he concludes that further research is "not really necessary'.

If parents do choose to treat fever at home, can they get the dose right on the basis of weighing the child on home scales? Costelloe et al in a 'spin-off' paper from the PITCH study ${ }^{3}$ (one those in Purssell's review) finds that they can, near enough.

If parents don't always get it right, then nor do doctors. Gordon et al sought to address this by designing and then assessing an e-learning package which teaches paediatric trainees to prescribe properly. They found it to be effective in improving and maintaining skills for 3 months, as assessed by re-testing. It remains to be seen whether these skills translate to improved patient care. See pages 1175, 1187 and 1191.

\section{Cystic fibrosis screening benefits extend to adult life}

For three decades the debate about universal neonatal screening for cystic fibrosis has raged. Earlier studies were not convincing about the benefits, but as evidence accumulated the argument in favour was won. New South Wales was one of the first places to introduce it in the early 1980s, and they are thus in a position to report its effectiveness as their patients become adults. Dijk et al report a historical cohort comparison of those diagnosed 3 years before and 3 years after the introduction of screening. The earlier benefits they described (on spirometry, body mass index (BMI) and survival) become, if anything, more pronounced as they reach the age of transition to adult care at around 17. Although it is a historical comparison rather than a RCT, it seems unlikely that improvements in treatment over a few years would account for all the differences. See page 1118.

\section{Season's greetings}

We are sometimes asked why Archives does not devote its December issue to 'fun' items, like the BMJ. The answer is that as a monthly rather than weekly publication, even if we had the material we could not afford the space. Authors of good papers rejected on priority would be understandably upset to see precious pages devoted to 'trivia'. However, we make one concession: the letter from Brown and Brown should amuse anyone whose jaw has dropped when told of the name parents have given their new baby. See page 1206.

We wish a peaceful and convivial festive season to all readers.

\section{REFERENCES}

1. Kendrick D, Stewart J, Smith S, et al. Randomised controlled trial of thermostatic mixer valves in reducing bath hot tap water temperature in families with young children in social housing. Arch Dis Child 2011:96:232-9.

2. James-Ellison M, Barnes P, Maddocks A, et al. Social health outcomes following thermal injuries: a retrospective matched cohort study. Arch Dis Child 2009;94:663-7.

3. Hay AD, Costelloe C, Redmond NM, et al. Paracetamol plus ibuprofen for the treatment of fever in children (PITCH): randomised controlled trial. BMJ 2008;337:a1302. 